\title{
Impact of balloon-assisted enteroscopy on the diagnosis and management of suspected and established small-bowel Crohn's disease
}

Authors

Institution
Udayakumar Navaneethan, John J. Vargo, K. V. Narayanan Menon, Madhusudhan R. Sanaka, Chung-Jyi Tsai

Department of Gastroenterology and Hepatology, Cleveland Clinic Lerner College of Medicine, Cleveland, Ohio, United States submitted 14. March 2014 accepted after revision 10. June 2014

\section{Bibliography}

Dol http://dx.doi.org/ 10.1055/s-0034-1377522

Published online: 12.11.2014 Endoscopy International Open 2014; 02: E201-E206

(c) Georg Thieme Verlag KG Stuttgart · New York E-ISSN 2196-9736

\section{Corresponding author}

\section{Udayakumar}

\section{Navaneethan, MD}

Department of

Gastroenterology and

Hepatology

Cleveland Clinic

Cleveland Clinic Lerner College of Medicine

9500 Euclid Avenue

Cleveland

Ohio 44195

United States

Fax: +1-216-444-6305

udhaykumar81@gmail.com
Background and aims: The role of recently developed balloon-assisted enteroscopy (BAE) in small-bowel Crohn's disease (CD) is not well established. The purpose of this study is to determine the clinical impact of BAE on patients with suspected and established small-bowel CD.

Methods: This study included 22 patients (group A) with suspected small-bowel CD and 43 patients (group B) with established small-bowel CD with or without previous surgery, who underwent $\mathrm{BAE}$, in a prospective BAE registry of a US academic medical institution. All underwent abdominal imaging studies including computed tomography $(\mathrm{CT})$ or magnetic resonance (MR) enterography before BAE. The main outcome measurements were diagnostic yield and clinical outcomes.

Results: In total, 78 BAE procedures were carried out in 65 patients. In group $A(n=22,25$ BAE procedures), enteroscopy led to a diagnosis of $C D$ in

\section{Introduction}

\section{$\nabla$}

Crohn's disease (CD) is a chronic inflammatory bowel disease characterized by a disabling course and transluminal inflammation which may involve small and large bowels [1]. In the past, the clinical goal of medical treatment was to achieve clinical remission. However, recent studies have reported better outcomes with mucosal healing, and this has now become the main goal of medical treatment [2]. Small-bowel capsule endoscopy (CE) is a very useful non-invasive tool for evaluating intestinal mucosal lesions in patients with $C D$ with small-bowel involvement. However, it lacks the capacity for a tissue diagnosis and for endoscopic treatment when it is needed [3]. Retention of the capsule endoscope, caused by small-bowel luminal strictures which often exist in patients with $C D$, is a major concern even when patency capsule endoscopy is available $[4,5]$. six patients $(27.3 \%)$. Non-steroidal anti-inflammatory drug-related enteropathy was diagnosed in three patients (13.6\%), whereas no lesions were found in the remaining 13 patients. In group $B(n=43,53$ BAE procedures) enteroscopy revealed active intestinal inflammation with ulcers and/or luminal stenosis in 18 patients (41.9\%), which led to a change and escalation of medical therapy. Five patients without active ulcers underwent successful dilation of small-bowel strictures with resulting resolution of obstructive symptoms. Of the 78 BAE procedures, two patients $(2.6 \%)$ had bleeding complications which were successfully treated conservatively. One patient (1.3\%) underwent surgery due to procedurerelated perforation.

Conclusions: The use of BAE may help improve management in patients with suspected and established small-bowel CD.

Balloon-assisted enteroscopy (BAE) has recently been developed for managing small-bowel diseases, and includes double balloon enteroscopy (DBE) and single balloon enteroscopy (SBE) systems. DBE has gained widespread acceptance and is the most established deep enteroscopy technique [6-16]. SBE and spiral enteroscopy (SE) are recently introduced techniques in endoscopic evaluation of the small bowel [17-19]. As compared with CE, BAE allows tissue biopsies for histopathology and therapeutic interventions including dilation of strictures [20]. Small-bowel strictures affect more than one-third of patients with $\mathrm{CD}$ and often cause intestinal obstruction leading to hospitalization and surgery [21]. The introduction of BAE provides a potential therapeutic alternative to surgery for $C D$ patients with small-bowel strictures.

At present, the role of BAE in patients with smallbowel CD is still not well established. The available reports to date are sparse and mostly have 
examined the utility of DBE and SBE individually in small series [22-25]. The purpose of this study was to assess the diagnostic yield and clinical impact of BAE in suspected and established small-bowel CD.

\section{Materials and methods}

$\nabla$

We reviewed our prospectively maintained BAE registry. This included DBE and SBE procedures on patients referred to the Cleveland Clinic between January 2005 and January 2012 for the investigation of small-bowel diseases. The database included patient demographics, findings of conventional endoscopy and radiological imaging, indications from procedures, findings from enteroscopy, and procedure-related complications. All patients provided informed consent for the enteroscopy procedures. The BAE registry was approved by the Cleveland Clinic Institutional Review Board. The indications of small-bowel evaluation in $C D$ were: (1) to achieve a definite diagnosis in patients with symptoms and signs indicative of $\mathrm{CD}$, but with inconclusive results from conventional endoscopy (esophagogastroduodenoscopy (EGD) and ileocolonoscopy), CE, and radiological cross-sectional imaging studies; (2) to assess disease activity and extent in uninvestigated $\mathrm{CD}$; (3) to investigate the cause of anemia or obscure gastrointestinal bleeding in $\mathrm{CD}$; $(4)$ to confirm and to treat small-bowel strictures visualized on radiological imaging; (5) to evaluate the extent and activity of $C D$ in postoperative patients deemed at high risk of CE retention. Before the BAE procedures, all patients underwent cross-sectional small-bowel imaging with contrast-enhanced computed tomographic (CT) enterography or magnetic resonance (MR) enterography, which evaluated the pattern of contrast enhancement, involvement of bowel segments, and stricture definition. For the study, we classified patients as suspected or established small-bowel CD [24], and investigated the utility of BAE in these patients.

Inclusion criteria for the analysis were antegrade and retrograde enteroscopy for suspected small-bowel CD after negative EGD and ileocolonoscopy or for characterization of small-bowel pathology detected by CE and/or other diagnostic imaging studies. Exclusion criteria for BAE included known large esophageal varices, fresh abdominal surgical stoma, medical instability, and inability to provide informed consent. Patients who underwent intraoperative enteroscopy were excluded.

The diagnosis of established $\mathrm{CD}$ was made based on endoscopic examination as well as from compatible histological examination. Presence of granulomas, patchy distribution of inflammation with skip lesions, longitudinal deep ulcers, presence of small-bowel involvement, presence of fistulizing disease and small-bowel strictures were taken as evidence of CD and classified based on published guidelines. All patients with isolated small-bowel CD had involvement of the ileum diagnosed based on ileocolonoscopy [26].

All procedures were performed by four experienced enteroscopists who had previous experience with BAE and advanced therapeutic endoscopy training. Office consultation and a history and physical examination with supporting laboratory studies were obtained before the procedures and assessed by the enteroscopist to determine the appropriateness of enteroscopy as the standard of care. The decision on using an antegrade or retrograde initial approach was primarily determined by clinical presentation. If the patient's clinical presentation was suggestive of upper small-bowel pathology on imaging or capsule endoscopy, then an antegrade approach was used for the study. DBE was routinely chosen for enteroscopy if the lesion of interest was beyond the distal jejunum. SBE was chosen if the lesion was proximal to the distal jejunum. If pathology was not reached with the initial insertion route, a tattoo was placed and the opposite anatomic approach was subsequently performed, as deemed clinically appropriate. All patients and their drivers were given standard discharge instructions and phone numbers to call to report any post-procedure problems or suspected complications.

Retrograde procedures were performed after standard bowel preparation. Antegrade procedures required no specific preparation apart from continuing to receive nothing by mouth for at least 8 hours before procedures. Patients were sedated with monitored anesthesia using propofol by an anesthesia provider as deemed appropriate. Fluoroscopy was used in selected cases, which depended on the preference of the performing enteroscopist and technical difficulty. Depth of insertion with DBE and SBE was measured in centimeters by counting the amount of small bowel traversed and cycles on withdrawal in 10-cm increments [27]. At the point of maximal depth of insertion, a tattoo was placed using SPOT ink as appropriate (GI Supply, Camp Hill, Pennsylvania, United States). Complications of enteroscopy in each procedure, if any, were recorded. A complication was defined as any event that changed the health status of a patient negatively, and that occurred during the 30-day period after BAE [28].

In this study, a stricture was defined by at least one of the following criteria, in addition to the clinical symptoms of intestinal obstruction: (1) enteroscopy showed an internal diameter of the small-bowel lumen estimated to be less than $10 \mathrm{~mm}$ or the enteroscope could not pass through the lesion; (2) a stricture was suggested or identified by other diagnostic modalities. Balloon dilation was performed through the endoscope with a controlled radial expansion wire-guided balloon dilatation catheter (Boston Scientific, Natick, Massachusetts, United States). The balloon was inflated with water to the pressure prescribed by the manufacturer for the size of the balloon. This pressure was maintained for 60 seconds or longer and this was repeated if required. The end point of dilation was the ability to pass the endoscope through the lesion. Balloon dilation might be repeated to treat the same lesion. In some patients with a deep open ulcer and/or a severe long stricture, dilation was not performed owing to a greater risk of intestinal perforation. Dilation was attempted later, if required, after inflammation had resolved following medical treatment with, for example, total parenteral nutrition or infliximab.

We reviewed all of the medications for the patients with a diagnosis of small-bowel CD before and after BAE. Escalation of medical treatment after BAE was defined as the addition of immunomodulators, including methotrexate, azathioprine, and 6-mercaptopurine, and/or the addition of biological agents, including infliximab, adalimumab, certolizumab, and natalizumab, to the existing baseline medical treatment.

DBE procedures were performed using the Fujinon endoscope system (EN-450T5, Fujinon Inc., Saitama, Japan). SBE procedures were performed using the SBE endoscope system (SIF-Q180, Olympus Optical, Tokyo, Japan).

Descriptive statistics were computed for all variables. These included means, medians, ranges for continuous variables, and frequencies and percentages for categorical variables. 


\section{Results}

$\nabla$

Two groups of patients were identified ( Table 1 ). The first group (group A) included 22 patients with suspected small-bowel CD (16 men; median age 46; interquartile range 39-64). All had symptoms and signs of chronic enteropathy and underwent standard diagnostic protocols. However, ileocolonoscopy and upper gastrointestinal endoscopy as well as histology had not revealed features diagnostic of $\mathrm{CD}$, whereas other chronic enteropathic disorders had been excluded. The second group (group B) included 43 patients ( 22 men; median age 41 ; interquartile range $32-54)$ with a previous diagnosis of small-bowel CD ( Tables 1 and 2).

Among the 22 patients in group A with suspected small-bowel $\mathrm{CD}, 25$ BAE procedures were performed ( $\bullet$ Fig. 1 ). There were no complications in all BAE procedures. All patients underwent
CT or MR enterography before BAE. Twenty patients in this group also underwent $\mathrm{CE}$ before BAE. Three patients had CE findings indicative of $C D$, while the $C E$ findings of the remaining 17 patients were deemed as nonspecific. CE was not performed in two patients due to the findings of luminal strictures on CT or MR enterography. CT or MR enterography revealed increased small-bowel wall thickness and post-contrast enhancement in 12 patients. With the findings of BAE and histopathology from enteroscopic biopsies, small-bowel CD was diagnosed in six patients, of whom one underwent successful balloon dilation of a jejunal stricture. Of the remaining 16 patients, non-steroidal anti-inflammatory drug-induced enteropathy was diagnosed in three patients, whereas no lesions were detected in 13 patients. These 13 patients had normal mucosa on BAE and no interventions were required.

\begin{tabular}{|c|c|c|}
\hline Variable & $\begin{array}{l}\text { Suspected CD } \\
\text { Group A, } n=22 \\
\text { ( } 25 \text { enteroscopies) }\end{array}$ & $\begin{array}{l}\text { Established CD } \\
\text { Group B, } n=43 \\
\text { ( } 53 \text { enteroscopies) }\end{array}$ \\
\hline Age, median (interquartile range), y & $46(39-64)$ & $41(32-54)$ \\
\hline Male gender, n (\%) & $16(72.7)$ & $22(51.2)$ \\
\hline \multicolumn{3}{|l|}{ Type of enteroscopy, n (\%) } \\
\hline DBE & $18(81.8)$ & $26(49.1)$ \\
\hline SBE & $7(18.2)$ & $27(50.9)$ \\
\hline \multicolumn{3}{|l|}{ Enteroscopy approach, n (\%) } \\
\hline Antegrade & $13(52)$ & $25(47.2)$ \\
\hline Retrograde & $12(48)$ & $28(52.8)$ \\
\hline \multicolumn{3}{|l|}{ Timing of enteroscopy, n (\%) } \\
\hline Morning & $12(48)$ & $25(47.2)$ \\
\hline Afternoon & $13(52)$ & $28(52.8)$ \\
\hline \multicolumn{3}{|l|}{ Associated significant EGD findings, $\mathrm{n}(\%)$} \\
\hline Erosive gastritis & $2(9.1)$ & $2(3.8)$ \\
\hline \multicolumn{3}{|l|}{ Associated significant colonoscopy findings, $n$ (\%) } \\
\hline Aphthous ulcers & $2(9.1)$ & $2(3.8)$ \\
\hline History of smoking, $n(\%)$ & $6(27.3)$ & $21(48.8)$ \\
\hline History of alcohol consumption, $\mathrm{n}(\%)$ & 0 & $1(2.3)$ \\
\hline Prior abdominal surgery, $\mathrm{n}(\%)$ & $19(86.4)$ & $36(83.7)$ \\
\hline Antegrade depth of insertion, estimated in $\mathrm{cm}$ & $159.7 \pm 120.5$ & $80.8 \pm 40.9$ \\
\hline Retrograde depth of insertion, estimated in $\mathrm{cm}$ & $45.7 \pm 29.9$ & $43.6 \pm 21.1$ \\
\hline Complications, $\mathrm{n}(\%)$ & 0 & $3(6.9)$ \\
\hline
\end{tabular}

Table 1 Clinical characteristics in suspected and established smallbowel Crohn's diseases.

DBE, double balloon enteroscopy; SBE, single balloon enteroscopy; EGD, esophagogastroduodenoscopy.

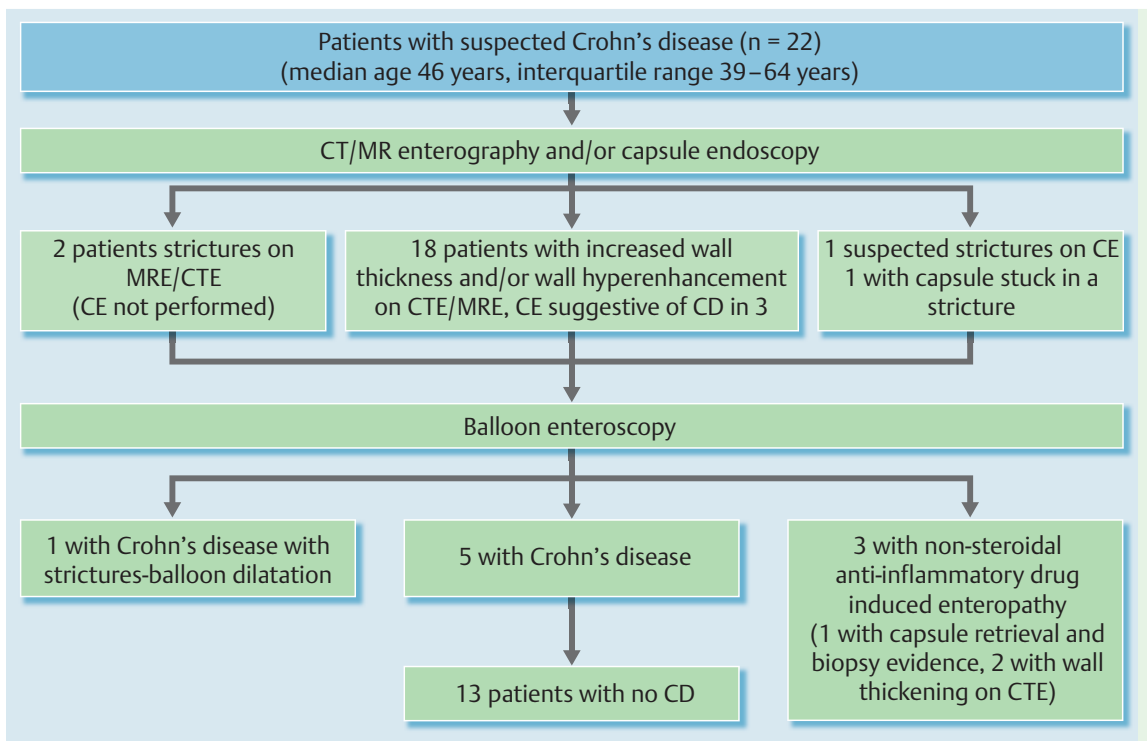

Fig. 1 Diagram summarizing the outcomes in patients with suspected small-bowel Crohn's disease undergoing balloon assisted enteroscopy (BAE). 


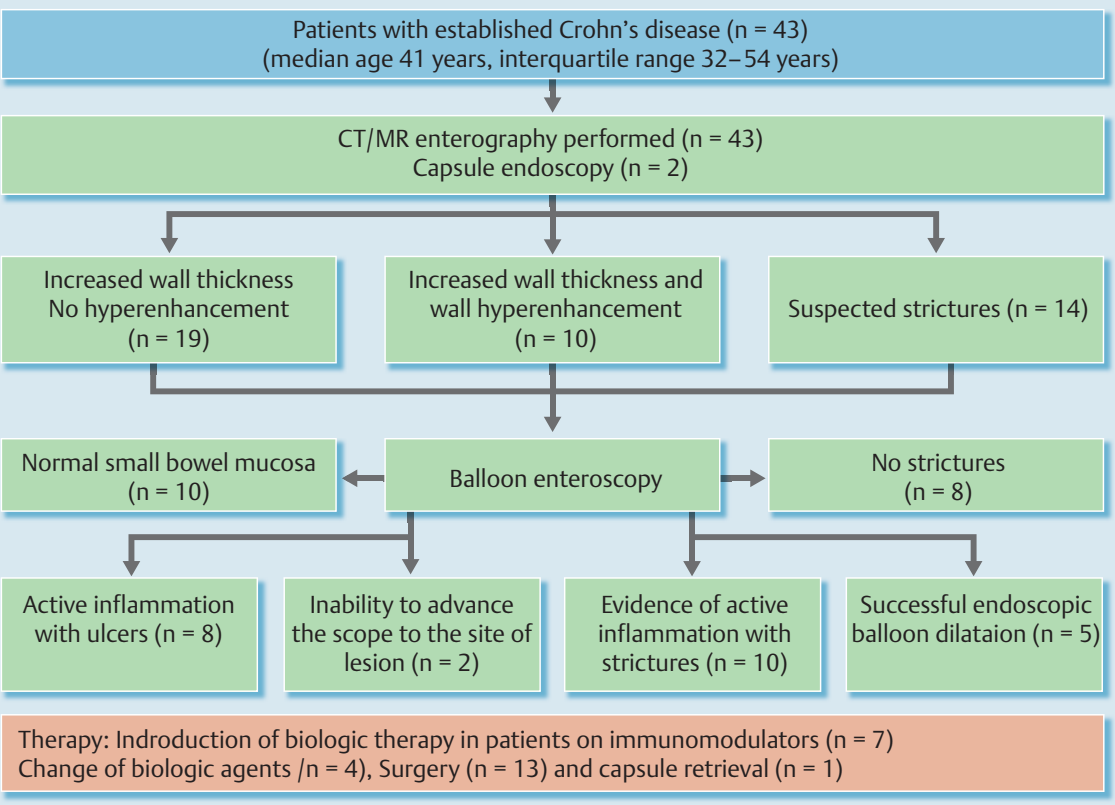

Fig. 2 Diagram summarizing the outcomes in patients with established small-bowel Crohn's disease undergoing balloon assisted enteroscopy (BAE).

We subsequently correlated the findings of CT or MR enterography with the BAE findings. Of the two patients with strictures on enterography, one patient had clear evidence of stricture on BAE, whereas the other patient had inflammation with biopsies suggestive of $\mathrm{CD}$. Twelve patients had increased wall thickness on enterography, of which two patients had NSAID enteropathy. The remaining 10 patients had a normal BAE. Six patients had wall thickening with enhancement, of which four patients had $\mathrm{CD}$ and the remaining two patients had a normal BAE. One patient with suspected CD on capsule endoscopy had a normal enterography and a normal BAE. One patient with capsule stuck in a stricture had evidence of stricture on enterography and BAE retrieved the capsule. The overall agreement of enterography with BAE findings was $8 / 22$ (36.4\%)

Among the 43 patients in group B with established CD, $53 \mathrm{BAE}$ procedures were performed ( Table 1 ). These patients underwent enteroscopy for further evaluation and management of lesions identified on diagnostic small-bowel imaging. All patients underwent CT or MR enterography before BAE, which revealed increased wall thickness in 19 patients, positive contrast enhancement in 10 patients, and stricture with pre-stenotic dilation in 10 patients and no pre-stenotic dilation in four patients ( $\bullet$ Fig. 2). CE was performed before BAE in only two patients because of the increased risk of capsule retention in the other patients in this group.

We subsequently correlated the findings of CT or MR enterography with the BAE findings in patients with established $C D$. Of the 14 patients with suspected strictures on enterography, eight patients had no stricture, while five patients had strictures and underwent balloon dilation of strictures through enteroscopy. One patient had strictures with inflammation which required medical management. Nineteen patients had increased wall thickness on enterography of which eight patients had active inflammation with ulcers, and in one patient, the enteroscope could not be advanced to the site of the lesion. The remaining 10 patients had a normal BAE. Ten patients had wall thickening with enhancement, of which nine patients had active inflammation with strictures whereas in the remaining one patient, the enteroscope could not be advanced to the area of interest. The overall agreement of enterography with BAE findings was $31 / 41$ (75.6\%)
Five patients without active intestinal ulceration underwent enteroscopic balloon dilation of small-bowel strictures with success ( $\bullet$ Fig.2). Fibrotic strictures were dilated with a through-thescope balloon ( Fig.3, Fig.4). All five patients but one had two sessions of balloon dilation to treat the strictures. Acute angulation was encountered in the small bowel during BAE procedures in two other patients in group $B$ because of adhesion-related tethering related to previous surgery, which prevented the enteroscope from reaching the strictures. BAE complications were encountered in three patients in group B with established smallbowel $\mathrm{CD}$. Perforation was present in one patient, and required immediate surgery. Two other patients had bleeding complications. Of these, one required hospitalization for treatment and transfusion, and the other was successfully treated with a local enteroscopic epinephrine injection at the bleeding lesion. Both bleeding episodes subsided with continued conservative management.

With the findings from the BAE investigation, seven patients who were on azathioprine subsequently had step-up treatment with biologics. Active inflammatory $\mathrm{CD}$ required an escalation in medical treatment ( $\bullet$ Fig.5). In the patients who were already on biologics, four had a change in biological agents from infliximab to adalimumab ( $\bullet$ Fig. 2). Seven patients elected to under-

Table 2 Characteristics of patients with established Crohn's disease.

\begin{tabular}{lc} 
Variable $(\mathbf{n}=\mathbf{4 3})$ & Number (\%) \\
\hline $\begin{array}{l}\text { Extent of CD } \\
\text { Small-bowel CD }\end{array}$ & $14(32.6)$ \\
Ileo-colonic CD & $26(60.5)$ \\
Ileo-colonic and duodenum CD & $3(6.9)$ \\
\hline Phenotype of CD & \\
$\quad$ Stricturing CD & $32(74.4)$ \\
Fistulizing CD & $11(25.6)$ \\
\hline 5-Aminosalicylate use & $10(23.3)$ \\
\hline Corticosteroid use & $17(39.5)$ \\
\hline Azathioprine/6-mercaptopurine use & $12(27.9)$ \\
\hline Biologics use & $20(46.5)$ \\
\hline Duration of CD, median (range), y & $8(1-14)$
\end{tabular}

$\mathrm{CD}$, Crohn's disease. 


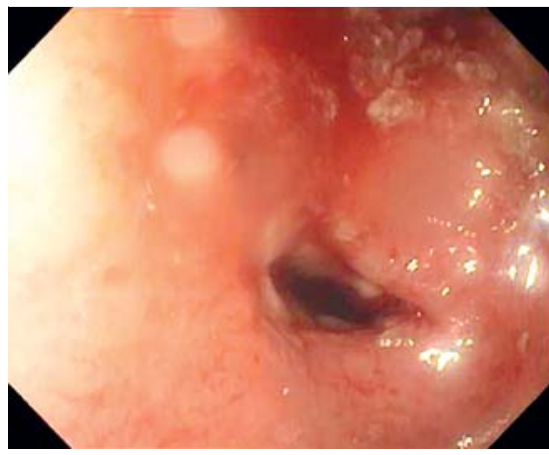

Fig. 3 Fibrotic stric ture from Crohn's disease in the mid-jejunum.

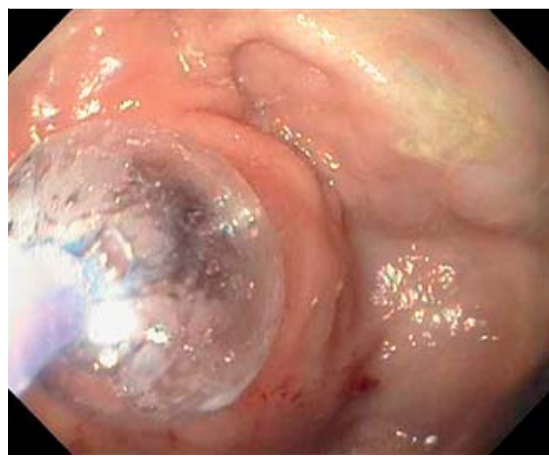

Fig. 4 Treatment of the stricture in the mid-jejunum by balloon assisted enteroscopy (BAE) and through-thescope balloon dilation.

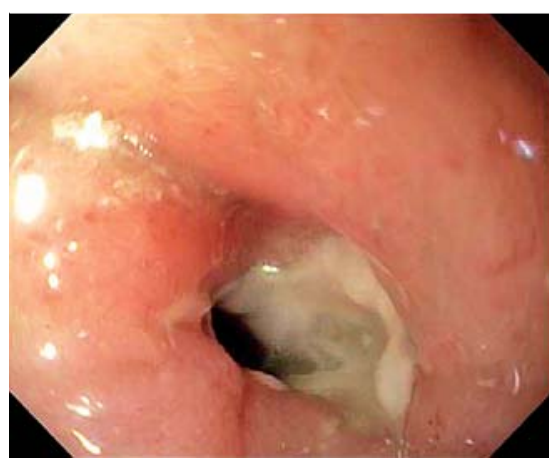

Fig. 5 Active inflammatory stricture from Crohn's disease in the ileum which required an escalation in medical treatment.

go surgery after BAE (two were on biologics, and five declined escalation in medical treatment). Of the 11 patients who received escalation in medical treatment after BAE, five required surgery after a median follow-up of 8 months, whereas the other six remained in clinical remission after a median follow-up of 10 months.

\section{Discussion}

$\nabla$

This report has shown that the use of BAE improves the clinical management of small-bowel CD. In patients with a diagnosis of small-bowel CD after BAE, adjustment of medical therapy resulted in clinical improvement. Among those who underwent therapeutic balloon dilation procedures, resolution of obstructive symptoms was achieved, which demonstrated that therapeutic BAE may be a valid alternative to surgery. This study showed that BAE is useful in the diagnosis and treatment of small-bowel lesions in $\mathrm{CD}$ patients.

Small-bowel involvement in CD is often associated with a complicated disease course including surgery $[21,29,30]$. Owing to the relapsing nature of $\mathrm{CD}$, it is important to avoid surgical resections as much as possible. Both $\mathrm{CE}$ and BAE have been used for endo- scopic evaluation of the small bowel to establish a diagnosis of Crohn's disease [31]. In our study, enteroscopy appears to be very useful for diagnosing small-bowel $C D$ when the findings of EGD and ileocolonoscopy are inconclusive. With the capability of obtaining histopathology in the small bowel for evaluation, BAE can help establish a diagnosis of small-bowel CD and, hence, direct appropriate treatment.

In patients with established $\mathrm{CD}$, previous studies have shown that both CE and cross-sectional enterography, either CT or MR, are useful diagnostic tools to investigate small-bowel involvement, especially when compared with small-bowel followthrough radiography and ileocolonoscopy [4,5,32-38]. However, CE can be associated with capsule retention in up to $7 \%$ of patients with small-bowel lesions [39]. In addition, incomplete small-bowel visualization was reported in up to $30 \%$ of CE [40]. Although CT and MR enterography can detect inflammation in the small bowel, BAE has the additional advantages of taking biopsies for histological evaluation to diagnose early mucosal disease and performing therapeutic dilation of intestinal luminal strictures.

We observed that $C D$ patients with small-bowel lesions benefited from adjustment of medical therapy following enteroscopy. Adjusting medical treatment resulted in clinical improvement in most patients. Although we did not perform follow-up enteroscopy to evaluate mucosal healing, improvement of abdominal symptoms had been observed in these patients. In the majority of our patients with small-bowel lesions confirmed by BAE, biological therapy was initiated, intensified or altered, resulting in clinical improvement, which demonstrated an additional benefit of treatment with these biological agents in this particular patient group with a complicated disease phenotype.

In this study, we also observed that BAE was effective in managing small-bowel CD strictures. The management of symptomatic small-bowel CD-associated strictures is challenging because of the generally poor response to medical therapy and the high rate of recurrence after surgical resection. Direct visualization of strictures during BAE procedures allowed the differentiation of patients with active inflammation or ulceration within the strictured bowel segments, who may benefit from medical treatment, from those with tight fibrotic strictures who may need endoscopic or surgical treatment. Our study showed that therapeutic BAE is effective in treating small-bowel strictures and may avoid surgery in selected patients.

There are limitations in this study. First, it was a retrospective analysis, however, the data were collected prospectively as patients were treated. The study population was recruited from a subspecialty tertiary referral center and only included patients in whom small-bowel mucosal activity was suspected. Followup enteroscopic evaluation to assess mucosal healing was not routinely performed after medical therapy, because clinical improvement itself was deemed sufficient to not pursue additional invasive investigations in these patients. The depth of maximal insertion was determined by estimation of the distance traversed into the small bowel and may not represent accurate scientific measurements. Carbon dioxide insufflation was not used in this study for comparison. Nonetheless, this is one of the largest studies on small-bowel CD patients, and showed that BAE improves the diagnosis and management of these patients.

Despite the fact that conventional cross-sectional radiological imaging and CE permit noninvasive exploration of the small bowel, the new BAE tools have enhanced our ability to manage small-bowel $\mathrm{CD}$ by allowing histological evaluation of small- 
bowel mucosa and, thus, the differentiation from other inflammatory intestinal disorders. Moreover, therapeutic BAE provides an invaluable nonsurgical means of treating small-bowel strictures.

In conclusion, our study has demonstrated the clinical usefulness of BAE in patients with suspected or established small-bowel CD. It allows a definite diagnosis of small-bowel CD when the earlier diagnosis was uncertain and improves clinical management and outcomes in patients with established small-bowel CD.

Competing interests: Dr John J. Vargo is on the speakers' bureau of Boston Scientific and is a consultant for Olympus. Drs Udayakumar Navaneethan, K. V. Narayanan Menon, Madhusudhan R. Sanaka and Chung-Jyi Tsai have no conflicts of interest or financial ties to disclose.

\section{References}

1 Baumgart DC, Sandborn WJ. Inflammatory bowel disease: clinical aspects and established and evolving therapies. Lancet 2007; 369: $1641-1657$

2 Pineton de Chambrun G, Peyrin-Biroulet L, Lémann M et al. Clinical implications of mucosal healing for the management of IBD. Nat Rev Gastroenterol Hepatol 2010; 7: 15-29

3 Mow WS, Lo SK, Targan SR et al. Initial experience with wireless capsule enteroscopy in the diagnosis and management of inflammatory bowel disease. Clin Gastroenterol Hepatol 2004; 2: 31-40

4 Buchman AL, Miller FH, Wallin A et al. Videocapsule endoscopy versus barium contrast studies for the diagnosis of Crohn's disease recurrence involving the small intestine. Am J Gastroenterol 2004; 99: 2271 2277

5 Kornbluth A, Legnani P, Lewis BS. Video capsule endoscopy in inflammatory bowel disease. Inflamm Bowel Dis 2004; 10: 278 - 285

6 Yamamoto $H$, Sekine $Y$, Sato $Y$ et al. Total enteroscopy with a nonsurgical steerable double-balloon method. Gastrointest Endosc 2001; 53: 216-220

7 Ell C, May A. Mid-gastrointestinal bleeding: capsule endoscopy and push-and-pull enteroscopy give rise to a new medical term. Endoscopy 2006; 38: $73-75$

8 Yamamoto $\mathrm{H}$, Kita $\mathrm{H}$, Sunada $\mathrm{K}$ et al. Clinical outcomes of double balloon endoscopy for the diagnosis and treatment of small-intestinal diseases. Clin Gastroenterol Hepatol 2004; 2: 1010-1016

9 Heine GD, Hadithi M, Groenen MJ et al. Double-balloon enteroscopy: indications, diagnostic yield, and complications in a series of 275 patients with suspected small-bowel disease. Endoscopy 2006; 38: 42 48

10 Monkemuller K, Fry LC, Neumann $H$ et al. Diagnostic and therapeutic utility of double balloon endoscopy: experience with 225 procedures. Acta Gastroenterol Latinoam 2007; 37: 216-223

11 May A, Nachbar L, Schneider M et al. Prospective comparison of push enteroscopy and push-and-pull enteroscopy in patients with suspected small-bowel bleeding. Am J Gastroenterol 2006; 101: 2016-2024

12 Sun B, Rajan E, Cheng S et al. Diagnostic yield and therapeutic impact of double-balloon enteroscopy in a large cohort of patients with obscure gastrointestinal bleeding. Am J Gastroenterol 2006; 101: 2011 - 2015

13 Mehdizadeh S, Ross A, Gerson L et al. What is the learning curve associated with double-balloon enteroscopy? Technical details and early experience in 6 U.S. tertiary care centers Gastrointest Endosc 2006; 64: $740-750$

14 Tanaka S, Mitsui K, Yamada Y et al. Diagnostic yield of double-balloon endoscopy in patients with obscure GI bleeding. Gastrointest Endosc 2008; 68: 683-691

15 Madisch A, Schmolders J, Bruckner $S$ et al. Less favorable clinical outcome after diagnostic and interventional double balloon enteroscopy in patients with suspected small-bowel bleeding? Endoscopy 2008; 40: $731-734$

16 Gross $S A$, Stark ME. Initial experience with double-balloon enteroscopy at a U.S. center. Gastrointest Endosc 2008; 67: 890-897

17 Tsujikawa T, Saitoh $Y$, Andoh A et al. Novel single-balloon enteroscopy for diagnosis and treatment of the small intestine: preliminary experiences. Endoscopy 2008; 40: $11-15$
18 Kawamura $T$, Yasuda $K$, Tanaka $K$ et al. Clinical evaluation of a newly developed single-balloon enteroscope. Gastrointest Endosc 2008; 68: $1112-1116$

19 Buscaglia JM, Dunbar KB, Okolo PI3rd et al. The spiral enteroscopy training initiative: results of a prospective study evaluating the Discovery SB overtube device during small bowel enteroscopy. Endoscopy 2009; 41: 194-199

20 Melmed GY, Lo SK. Capsule endoscopy: practical applications. Clin Gastroenterol Hepatol 2005; 3: 411-422

21 Van Assche G, Rutgeerts P. Medical management of postoperative recurrence in Crohn's disease. Gastroenterol Clin N Am 2004; 33: 347- 360

22 Murphy SJ, Kornbluth A. Double balloon enteroscopy in Crohn's disease: where are we now and where should we go? Inflamm Bowel Dis 2011; 17: 485-490

23 Mensink PB, Aktas $H$, Zelinkova $Z$ et al. Impact of double-balloon enteroscopy findings on the management of Crohn's disease. Scand J Gastroenterol 2010; 45: 483-489

24 Di Nardo G, Oliva S, Aloi M et al. Usefulness of single-balloon enteroscopy in pediatric Crohn's disease. Gastrointest Endosc 2012; 75: 80-86

25 Oshitani N, Yukawa T, Yamagami H et al. Evaluation of deep small bowel involvement by double balloon enteroscopy in Crohn's disease. Am J Gastroenterol 2006; 101: 1484-1489

26 Annese V, Daperno M, Rutter MD et al. European evidence based consensus for endoscopy in inflammatory bowel disease. J Crohns Colitis 2013; 7: $982-1018$

27 May A, Nachbar L, Schneider M et al. Push-and-pull enteroscopy using the double-balloon technique: method of assessing depth of insertion and training of the enteroscopy technique using the Erlangen EndoTrainer. Endoscopy 2005; 37: 66-70

28 Mensink PB, Haringsma J, Kucharzik T et al. Complications of double balloon enteroscopy: a multicenter survey. Endoscopy 2007; 39: 613-615

29 Fukumoto A, Tanaka S, Yamamoto $H$ et al. Diagnosis and treatment of small-bowel stricture by double balloon endoscopy. Gastrointest Endosc 2007; 66: 108 -S112

30 Silverstein MD, Loftus EV, Sandborn WJ et al. Clinical course and costs of care for Crohn's disease: Markov model analysis of a population based cohort. Gastroenterology 1999; 117: 49-57

31 World Organization of Digestive Endoscopy (OMED). the European Crohn's and Colitis Organization (ECCO). Role of small-bowel endoscopy in the management of patients with inflammatory bowel disease: an international OMED-ECCO consensus. Endoscopy 2009; 41: 618 637

32 Pasha SF, Leinghton JA. Enteroscopy in the management of Crohn's disease. Gastrointest Endosc Clin N Am 2009; 19: 427-444

33 Herfarth HH, Long MD. Capsule and balloon endoscopy: when are they really needed in patients with inflammatory bowel diseases? Dig Dis 2010; 28 : 439-444

34 Zisman TL, Rubin DT. Novel diagnostic and prognostic modalities in inflammatory bowel disease. Med Clin N Am 2010; 94: 155-178

35 Triester SL, Leighton JA, Leontiadis GI et al. A meta-analysis of the yield of capsule endoscopy compared to other diagnostic modalities in patients with nonstructuring small bowel Crohn's disease. Am J Gastroenterol 2006; 101: 954-964

36 Ochsenkühn T, Herrmann K, Schoenberg SO et al. Crohn's disease of the small bowel proximal to the terminal ileum: detection by MR enteroclysis. Scand J Gastroenterol 2004; 39: 953-960

37 Herrmann KA, Zech CJ, Michaely HJ et al. Comprehensive magnetic resonance imaging of the small and large bowel using intraluminal dual contrast technique with iron oxide solution and water in magnetic resonance enteroclysis. Invest Radiol 2005; 40: 621 - 629

38 Chiorean MV, Sandrasegaran K, Saxena R et al. Correlation of CT enteroclysis with surgical pathology in Crohn's disease. Am J Gastroenterol 2007; 102: $2541-2550$

39 Matsumoto T, Esaki M, Moriyama T et al. Comparison of capsule endoscopy and enteroscopy with the double-balloon method in patients with obscure bleeding and polyposis. Endoscopy 2005; 37: 827-832

40 Chong AK, Chin BW, Meredith CG. Clinically significant small-bowel pathology identified by double-balloon enteroscopy but missed by capsule endoscopy. Gastrointest Endosc 2006; 64: 445-449 